\title{
A method to evaluate equitable accessibility: combining ethical theories and accessibility-based approaches
}

\author{
Karen Lucas $\cdot$ Bert van Wee $\cdot$ Kees Maat
}

Published online: 3 March 2015

(C) The Author(s) 2015. This article is published with open access at Springerlink.com

\begin{abstract}
In this paper, we present the case that traditional transport appraisal methods do not sufficiently capture the social dimensions of mobility and accessibility. However, understanding this is highly relevant for policymakers to understand the impacts of their transport decisions. These dimensions include the distribution of mobility and accessibility levels over particular areas or for specific population groups, as well as how this may affect various social outcomes, including their levels of participation, social inclusion and community cohesion. In response, we propose a method to assess the socially relevant accessibility impacts (SRAIs) of policies in some of these key dimensions. The method combines the use of underlying ethics principles, more specifically the theories of egalitarianism and sufficientarianism, in combination with accessibility-based analysis and the Lorenz curve and Gini index. We then demonstrate the method in a case study example. Our suggestion is that policymakers can use these ethical perspectives to determine the equity of their policies decisions and to set minimum standards for local transport delivery. This will help them to become more confident in the development and adoption of new decision frameworks that promote accessibility over mobility and which also disaggregate the costs and benefits of transport policies over particular areas or for specific under-served population groups.
\end{abstract}

Keywords Accessibility - Ethics - Equity - Transport-related social exclusion · Egalitarianism $\cdot$ Sufficientarianism

\footnotetext{
K. Lucas

Institute for Transport Studies, University of Leeds, Leeds LS2 9JT, UK

e-mail: K.Lucas@leeds.ac.uk
}

B. van Wee $(\bowtie) \cdot$ K. Maat

Transport and Logistics Group, Delft University of Technology, PO Box 5015, 2600 GA Delft, The Netherlands

e-mail: g.p.vanwee@tudelft.nl

K. Maat

e-mail: c.maat@tudelft.nl 


\section{Introduction}

Transport policies generally aim to improve accessibility and reduce the negative impacts of motorised transport. Most countries have adopted cost-benefit analysis (CBA) as the preferred evaluation methodology across all aspects of transport decision-making (Hayashi and Morisugi 2000; Bristow and Nellthorp 2000; Grant-Muller et al. 2001; Thomopoulos and Grant-Muller 2013). However, CBA generally ignores the distribution effects of such decisions, such as how they affect different regions and/or social groups. If policies explicitly aim to reduce inequalities in accessibility, or might have unequal (and potentially disproportionate) negative impacts for certain social groups, this can be problematic.

Some transport policies directly aim to reduce transport-related social exclusion through the introduction of new transport services and a variety of supporting measures (Department for Transport 2006). People experiencing social exclusion are rarely willing (or able) to pay the full cost of these new services and so they must be subsidised through the public purse. As such, evaluating these type of policies using a CBA framework, which assesses people's willingness to pay (WTP), is not generally useful. This suggests there is a need for the widespread adoption of a robust method for policy-makers to easily and effectively evaluate the increasingly important social policy outcomes of transport policy.

This paper presents a method which combines the two ethical theories of egalitarianism and sufficientarianism with an accessibility-based approach to develop a measure of the 'socially relevant accessibility impacts' (SRAIs) of transport policies. We first briefly present current transport appraisal methods and their limitations in terms of evaluating the potential social equity and inclusion implications of transport policies ("Assessment of equity within current transport appraisal methods" section) in the assessment of SRAIs. Section "Using egalitarianism and sufficientarianismtheories to evaluate the equity and accessibility aspects of transport policy decisions" describes two ethical theories to evaluate equity and accessibility aspects of policies. Section "Amethod for SRAIs based upon the contrasting principles of egalitarianism and sufficientarianism" develops the method and presents indicators for applying the SRAI assessments based on these theories. Section "Application of the method" applies the method in a case study. Section "Critical discussion and implications of the SRAI method for future transport policy decisionmaking" critically reflects on some of the limitations of the method and the implication for future transport policy decision-making. Finally, Section "Concluding remarks" presents some concluding remarks.

\section{Assessment of equity within current transport appraisal methods}

In this section of the paper, we discuss two of the most popular current methods for evaluating the impacts of transport policy decisions: (i) cost benefit analysis (CBA) and (ii) multi-criteria analysis (MCA). Our intention here is not to discredit the value of these approaches where they are 'fit for purpose', but rather to describe how their widespread adoption has served to preference particular aspects of the transport provision, such as journey-time savings or operational efficiencies, over other less tangible or unknown aspects, such as whether it promotes equality of opportunity and social inclusion. 
Cost benefit analysis

One of the main advantages of CBA is that it is designed to weigh-up the pros and cons of different candidate policy options (using standardised monetary values) and to summarise the results using directly comparable measures, such as benefits minus costs, or the benefitto-cost ratio. There are two further important explanations for its prominent role and popularity in the ex-ante evaluations of new transport infrastructure projects (and other policy options).

Firstly, in many cases most of the relevant costs and benefits are relatively wellknown and quantifiable: costs include investment, maintenance and operation costs. In transport CBA, the most important benefits are expressed as travel time savings, both for travellers and freight transport, and can be monetised using relatively straightforward and standardised measures of Willingness-To-Pay (WTP) based on long-standing and well-accepted studies of stated preference. Secondly CBA is seen as being relatively 'value neutral' compared to its main competitor Multi Criteria Analysis (MCA), which requires either policy experts or other stakeholders to determine the value of different system or service attributes (Van Wee 2007). We discussed this further in section "Multi-criteria analysis" below.

Despite its popularity, traditional CBA has come under regular criticism within the transport literatures for a variety of reasons (see, for example, Thomopoulos et al. 2009; Grant-Muller et al. 2001; Sagoff 1988; Gardiner 2006; Van Wee 2012). A particular criticism of $\mathrm{CBA}$ of relevance to this paper is that it tends not to address the equity effects and other ethically important implications of policy options, e.g. in the area of social exclusion (Jones and Lucas 2012). This is despite wide recognition that 'sound' policies should meet three criteria: (i) effectiveness, (ii) efficiency and (iii) equity (e.g. Young and Tilley 2006).

In fact, a CBA can report on distributional effects, for example Annex 5 of the Treasury's Green Book (HM Treasury, 2014) considers using utility-based weights to reflect differences between beneficiaries (and those who bear the costs) based on their levels of income, but these are not usually attributed a financial value and consequently have no impact on the final outcome of the CBA. The concept of Social Welfare Functions (SWF) aims to measure preferences for social preferences (e.g. Arrow 1963). However, there is no generally accepted way to measure individual well-being (Nyborg 2000). In the case of policies that are designed to have an impact on the levels of social equity and inclusion, it is obvious that policy makers need to specifically understand which groups and areas benefit and who loses out (Social Exclusion Unit 2003).

\section{Multi-criteria analysis}

Multi-criteria analysis (MCA) has increasingly been adopted within transport policy and project appraisals to either replace or supplement CBA in the light of some of these important above criticisms. An MCA provides an overview of the scores of candidate policy options based upon different criteria. These can be expressed using any indicator of measurement (i.e. in monetary terms, using quantitative levels of an indicator, or ordinal scores such as $++,+, \mathrm{o},-,--)$. A final score of each policy is given based on weights of these different criteria and scores, which is often used for ranking the policy options. One of the key advantages of using an MCA approach is that each criterion can be measured and evaluated according to its own scale within a single evaluation framework. It is, therefore, possible to include, score and compare different (and non-monetary) values and 
set their value using a set weight for each criteria. Setting the weights is not value free (Eijgenraam et al. 2000) and there should be processes put in place to control for arbitrary and non-validated value judgements in this respect. Commonly, MCA assessments include the key social factors that are associated with increased mobility and/or accessibility, such as participation in new activities, health benefits and disbenefits and quality of life issues (Hine and Mitchell 2001; Litman 2012).

A major disadvantage of MCA is the risk of double counting. For example, a new bus service may reduce travel times, as well as the level of social exclusion, but the latter is reduced as an outcome of the former. If the travel time reduction is given a certain weight and the reduced level of social exclusion another, there is overlap between the impact value of the two criteria because the reduced travel times of people after the implementation of the new bus service are not socially excluded anymore but are effectively counted twice. MCA also does not inherently identify the spatial or social distribution of impacts across different population groups and this needs to be specifically identified as a separate necessary step if there is a concern to protect or enhance the accessibility of particular 'at risk' groups.

Whichever methodological approach is preferred, CBA or MCA, each requires some judgements on the part of the evaluator in terms of how to measure and weight the various SRAIs. As there is no universally adopted method for calculating these impacts and this will always be highly dependent on the moral values, standards and norms of the society in question.

However, most policymakers remain largely oblivious to the underpinning ethical principles upon which their evaluation frameworks are based and so may overlook the inherent value biases within them. For example, the traditional ethical framing for transport policy evaluations via CBA is broadly based upon the principles of utilitarianism, which is an ethical theory within the wider family of consequentialism. Consequentialism and more specifically, 'act consequentialism': "is the claim that an act is morally right if and only if that act maximizes the good, that is, if and only if the total amount of good for all minus the total amount of bad for all is greater than this net amount for any incompatible act available to the agent on that occasion" (Stanford Encyclopaedia of Philosophy-http:// plato.stanford.edu/entries/consequentialism/). ${ }^{1}$

Although this framing can be useful for many areas of policy delivery, especially where the aim is to maximise the benefits of an investment for all members of society, it is not appropriate when there is specific aim to achieve greater equity from that investment. In an effort to address this shortfall, we next discuss the development of a method based upon two ethically-driven alternatives to utilitarianism, namely egalitarianism and sufficientarianism. We suggest that these alternative approaches more adequately justify policies that specifically aim to redistribute transport resources towards currently disadvantaged population groups and deprived areas.

\section{Using egalitarianism and sufficientarianism theories to evaluate the equity and accessibility aspects of transport policy decisions}

It is widely accepted within the literature that no policy decisions are entirely value-free and so it is important to be explicit about the value system that is in place when policy interventions are being developed and evaluated. The use of ethical principles to support

1 Accessed 8-8-2014. 
the values that are being promoted through public policies is not new but it is an issue that still concerns many transport professionals (see Martens et al. 2012 and Hyard 2012). Van Wee and Geurs (2011) offer a comprehensive discussion of the different ethical theories that are relevant to social exclusion. Our particular rationale for choosing the two contrasting theories of egalitarianism and sufficientarianismin this paper is that they help to demonstrate how different moral interpretations of fairness can result in the adoption of different policy measures. In Section "Application of the method", we come back to comparing the results based on each theory in our case study example.

\section{Egalitarian theories}

Egalitarians believe that society should treat all people equally (Sen 1992; 2009). Whereas the theory of utilitarianism upon which CBA is based tells us that we should maximise the sum of benefits for all people, John Rawls', A Theory of Justice (Rawls 1971), differs in two important respects. Firstly Rawls argues that for justice we should not focus on welfare but rather on the provision of what he labels 'primary social goods'. Secondly, he argues that we should strive for 'the greatest benefit of the least advantaged members of society' (the max-min principle (Kelly 2001, pp. 42-43).

Even though Rawls (to the best of our knowledge) never specifically identified accessibility as a primary social good, Van Wee and Geurs (2011) make a good case for doing so. Martens (2012) also does this within his discussion of Walter's 'Spheres of Justice' thesis. Here he finds that goods such as transport and accessibility have socially constructed meanings (similar to education and health care), which differs across different societies and social groups. These goods, therefore, have comparable rather than absolute values depending on the differential benefits and burdens they bring. As such, we need to understand the social meaning of the transport-good before we can determine its comparable value and whether this is being fairly distributed.

Using egalitarian theories to evaluate the equity of different transport policies justifies the case for moving away from journey-time savings as the primarily valuation method and towards (improved)accessibility to basic services (such as grocerystores and other daily activity-centres, such as work places, schools, and medical centres) (Van Wee and Geurs 2011). It also encourages a policy focus on equalising the relative level of accessibility between different social groups. From such a perspective, the benefits of providing a new bus service to improve the accessibility of unemployed young people to relevant employment opportunities would be valued more highly than the aggregate journey time savings of the whole population using that bus. To conclude, egalitarian theories are particularly useful to legitimate policy that aim for equality of accessibility.

\section{Sufficientarianism}

While egalitarian theories focus on differences between people, sufficientarianism assumes that everybody should be welloff up to a certain minimum threshold, which is 'sufficient' for fulfilling their basic needs and to guarantee their continued wellbeing. 'Weak sufficientarianism' suggests it is important to improve the well-being of those people who are below the minimum threshold. 'Strong sufficientarianism' tells that absolute policy priority should be given to the improvement of the well-being of these people; the lower their welfare, the more important is the policy priority (Meyer and Roser 2009; Wolf 2009).

The concept of sufficientarianism therefore provides an ethical justification for developing policies that provide a minimum threshold level of accessibility to key destinations. 
Below this level people are considered to be socially excluded (see Lucas 2012). For the ex-ante evaluation of the SRAI of different transport policy options a hybrid egalitarian and sufficientarian approach is needed, as we explain in the next section of the paper.

\section{A method for SRAIs based upon the contrasting principles of egalitarianism and sufficientarianism}

In the area of applied ethics, it is common to adopt qualitative rather than quantitative methods in order to come to a moral judgment about how to deal with a given policy problem. The evaluator identifies an ethical problem, next chooses a suitable ethical theory, and finally analytically comes to a set of moral conclusions. Whilst this helps to decide whether a given policy option is morally fair or not, it does not offer a measurement or a value with which to easily assess and compare the fairness of that option with another option.

In the case of transport decision-making, however, there has been a long and welldeveloped tradition of quantitative evaluation. It is, therefore, difficult to persuade policymakers tasked with the evaluation of different transport policy options to accept qualitative judgements of their contribution to SRAIs. This has been repeatedly demonstrated in the case of the UK, where the Department of Transport (2002) has made a considerable and repeated effort to develop quantitative measures of the social and distributional impacts of transport projects and policies (for the latest guidance see Department for Transport, WebTAG Guidance-Units A4.1 and A4.2, 2014). To this end, we next propose a quantitative method for the measurement of SRAI to fit with this general policy tradition.

\section{Calculating measures of accessibility}

Numerous authors have emphasised the importance of accessibility measures for assessing the social equity and distribution inclusion impacts of transport services (e.g. Social Exclusion Unit 2003; Hurni 2006; Preston and Raje 2007; Mackett et al. 2008; Páez et al. 2009; Currie et al. 2009). A broad definition of accessibility refers, not only to physical access to goods and services, but also the transport system itself in terms of its availability (including routing and scheduling), affordability, reliability and safety, as well as access to timetable information, etc.

Accessibility can either be calculated using place-based perspective (i.e. from one geographical location to another) or person-based measures (i.e. also considering personal characteristics, resources and capabilities, including time budgets, etc.). Neutens et al. (2010) have found person-based measures to be more conservative than place-based measures in terms of assessing the level of equity of service delivery.

In their review of the accessibility literature, Geurs and Van Wee (2004) ${ }^{2}$ identify four important components of accessibility that are important for policymakers to consider in their evaluations of the transport system:

\footnotetext{
${ }^{2}$ We changed the order of Geurs and van Wee to better emphasize the importance of components for a discussion on social exclusion. The order is (1) what do people need, (2) where are locations of activity needs, (3) how to get there, and finally (4) when to go there.
} 
1. The individual component reflects the needs (depending on age, income, educational level, household characteristics etc.), abilities (depending on people's physical condition, availability of travel modes etc.) and opportunities (depending on people's income, travel budget, educational level, etc.) of individuals. These characteristics influence a person's level of access to transport modes (e.g. being able to drive and borrow/use a car) and spatially distributed opportunities (e.g. have the skills or education to qualify for jobs near their residential area), and may strongly influence their total aggregate accessibility levels.

2. The land-use component reflects the land-use system, consisting of (a) the amount, quality and spatial distribution of opportunities supplied at each destination (jobs, shops, health, social and recreational facilities, etc.), (b) the demand for these opportunities at origin locations (e.g. where inhabitants live), (c) the confrontation of supply and demand for opportunities which may result in competition for activities with restricted capacity such as jobs, school vacancies and hospital beds (Van Wee et al. 2001).

3. The transportation component describes the transport system, expressed as the disutility experienced by an individual when covering the distance between an origin and a destination; included are the amount of time (travel, waiting, parking), costs (fixed and variable) and comfort-related variables (such as reliability, level of comfort, accident risk, etc.). This disutility partly results from the conflict between supply and demand of infrastructure capacity. The supply includes the location and characteristics of transport services (e.g. maximum travel speeds, service frequencies, safety, reliability, public transport timetables, travel costs, etc.). The demand for infrastructure can relate to both passenger and freight travel, but in this case only passenger travel is considered.

4. The temporal component reflects the temporal constraints, i.e. the availability of opportunities at different times of the day, and the time available for individuals to participate in certain activities (e.g. work, recreation). Note that this temporal component has enjoyed a rapid increase in popularity amongst academics in transportation and geography (e.g. Schwanen \& Kwan 2008) and usefully serves to illustrate that some social groups, particularly women, can become excluded from participation due to their work/home responsibilities and time budget constraints.

5. In addition, Lucas (2012) has suggested in the evaluation of transport-related social exclusion it is also important to consider a fifth cognitive component, which includes people ability to interact with the transport system. This would include experience of the transport system, confidence whilst travelling, people's travel horizons and other cultural factors. These factors are especially important when considering the needs of certain social groups, such as people with mental disabilities, low literacy skills or ethnic minority populations.

All five of these accessibility components can and will interact with each other in all directions, so that disadvantage in one dimension can potentially be compensated by creating advantage in another. For example, the disappearance of the last shops in a village (the land use dimension) can be compensated by better public transport options to travel to shopping areas in a nearby town (the transport dimension).

Use of the Gini index

Having identified appropriate measures of accessibility, the next step is to identify the distribution of accessibility between different social groups and/or areas. Some authors 
have previously proposed the Gini coefficient or index (Gini 1936) as the basis for transport equity evaluation (e.g. see Rietveld et al. 2007; Van Wee and Geurs (2011), others have proposed non-Gini-index based indicators that also use normalised scores (on a scale ranging from zero to one) (Thomopoulos and Grant-Muller 2013). We build upon those papers to present a method that shows how two (categories of) ethical theories, egalitarianism and sufficientarianism, can be explicitly linked to the Gini-index.

A major advantage of the Gini index over other equality indices is that it is scaleindependent (insensitive to changes in measurement, such as currency or price year in case of income-see Ramjerdi 2006). Note that even though the Gini index is scale independent, it does depend on the choice of the indicator. If, for example, a contour measure would be chosen such as the number of jobs accessible within $\mathrm{X}$ minutes by car, the value of $\mathrm{X}$ can make a big difference, both in terms of the Gini index as well as with respect to the evaluation of individual units (e.g. people,/households,/areas). To demonstrate the importance of $\mathrm{X}$ on an area level: a town centrally located between larger cities at a distance of 30 min could perform badly if $X$ has the value of 20, whereas it could perform very well if $\mathrm{X}$ has the value of 40 .

The Gini index is more widely accepted as a statistical measure of inequality, the dominant application being the distribution of income over the population of a country (e.g. Weymark 1981). The Lorenz curve is then used to show the cumulative distribution of income over the cumulative population ordered by increasing share of income. If, for example, $50 \%$ of the population with the lowest incomes together earns $15 \%$ of a countries' income, the Lorenz curve will be more bent compare to the situation in which this group would earn $30 \%$ of a countries' income. The Gini index is the area between the line of equal distribution and the Lorenz curve, divided by the triangle covering the $\mathrm{x}$-axe, the $y$-axe and the line of equal distribution. If all income in a country is allocated to only one person its value is 1 ; if all people have the same income it is 0 (see also below, Fig. 1).

González-Pérez et al. (2008) previously applied the Gini index in the health context in state and capital of Mexico and Wagner et al. (2009) have also applied it in the context of pharmacies in England. The index has also been previously used in the transportation context. For example, in their study of Ghent in Belgium, Neutens et al. (2010) used Gini scores to compare place-based and person-based measures of accessibility for men and women, Santos et al. (2008) used it in the context of accessibility evaluation of road network design, Tian et al. (2012) used it to evaluate efficiency and equity effects of ramp control schemes, Karlström and Franklin (2009) used it to evaluate equity effects of the Stockholm congestion pricing trial and Delbosc and Currie (2011) have used it to assess the social exclusion effects of public transport services in Melbourne, Australia. Van Wee (2011) and Van Wee and Geurs (2011) have argued the Gini index is applicable to evaluate accessibility and social exclusion effects. To our knowledge, the Gini index has not previously been explicitly linked with the principles of egalitarianism and sufficientarianism within the transport context.

The next two sections discuss how this can be achieved by linking these prinicples to accessibility indicators.

\section{Indicators of egalitarianism}

As identified above, if the aim is to assess the equality of accessibility then assessment is most appropriately based upon the principles of egalitarianism. In this instance, the people for which the accessibility indicator is calculated are recorded on the X-axis (see Fig. 1 above), and the cumulative distribution of this accessibility across the population is 
recorded on the Y-axis, resulting in the Lorenz curve of accessibility. The indicators could be any quantitative accessibility indicator (see Geurs and Van Wee 2004, for an overview), such as potential values, catchment areas or other indicators to measure access to basic services, ora space-time measure. It is important to realise that-unlike income-the sum of accessibility over all people does not represent an overall level of accessibility. In case of income the sum is equal to all income earned by all people and each currency unit (e.g. Euro) only counts once.

In the specific case of SRAI policies, accessibility should be measured for the activities with most relevance to this policy agenda (see Lucas 2012), including employment, education, health and welfare services, etc. (other indicators of accessibility could also be selected for the sake of evaluating more generalised equality in accessibility). The Gini score is then an indication of the level of inequality of the accessibility indicator and therefore is related to egalitarianism.

An alternative would be to measure journey times for all people living in a certain area, to a selection of destinations that are assumed to be most relevant from the SRAI perspective and next to calculate the index expressing the inequality of these journey times. Journey times can be analyses for each destination type separately, or for a set of destination types simultaneously.

Calculating the index for either accessibility or journey times can be undertaken (1) on an individual basis, (2) for different Socio-Economic Groups (SEGs) to be included in one calculation-these groups would then be the units of the X-axis (see Fig. 1), and (3) for different SEGs in a separate calculation. The third approach results in Gini scores per SEG. Not only is the evaluation for each SEG of interest, so is comparing the results per SEG, because this gives an impression of differences in the level of inequality between SEGs.

The impact of different policies on these different social groups would be evaluated by looking at changes in the index due to policies. However, strictly speaking, used in this way, the index is only measuring inequality and not social exclusion. To explicitly include a threshold value below which people are socially excluded we need to add the principles of sufficientarianism to the evaluation, as is now described.

Fig. 1 The Gini index and Lorenz curve applied to the concept of sufficientarianism

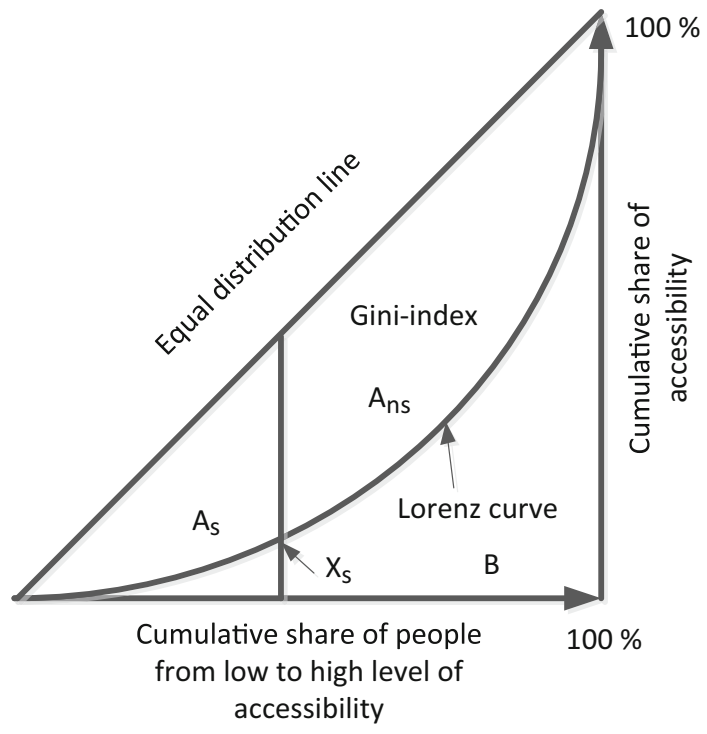


Indicators of sufficientarianism

In case of sufficientarianism, threshold values for accessibility need to be defined below which it can be assumed individuals will become 'socially excluded'. This is a methodological challenge in itself because the condition of being socially excluded is relational (i.e. dependent on the accepted norms of the societies in which people are located) and will, therefore, be both context- and person-specific (see Lucas 2012). As such, new empirical studies will be needed in order to establish the appropriate threshold values of accessibility for different population groups in different geographical contexts. In some countries (e.g. the UK see Lucas 2012) this research is already well advanced, whereas in others it has not even begun.

Related to this threshold issue, is whether minimum standards should be based on the opportunity to participate in activities or revealed levels of participation, as well as what range of activities should be considered and how far an element of personal choice should be accommodated. Another issue is at what critical point should policy intervene, i.e. what minimum level legitimates or necessitates policy intervention? This is a moral issue, and ultimately a political choice.

For the sake of simplicity, here we assume that we already have such a threshold value based on the UK indicators (Social Exclusion Unit 2003). This value can be converted to the slope of the Lorenz curve: at the right side of the point on the curve where the slope is above the threshold value (Xs) we find people whose access is above the threshold value, at the left side we find people we label as 'socially excluded'. Note that the slope of the curve expresses the accessibility level of the related unit (e.g. a person) on the X-axis. In other words, the increase in the $\mathrm{Y}$-axis as a result of shifting one unit more to the right on the $\mathrm{X}$-axis expresses the accessibility level of the related unit on the $\mathrm{X}$-axis.

The corresponding value on the $\mathrm{X}$-axis tells which percentage of the population is socially excluded. In addition we propose an indicator for the severity of social exclusion. We split area A into two parts: left versus right of the point Xs: the part of area A left of the point (As = socially excluded area, as opposed to Ans = not socially excluded area) divided by the triangle $(\mathrm{A}+\mathrm{B})$ is an indicator for the severity of social exclusion (see Fig. 1 above in which we visualise the Gini index for accessibility; journey times could be an alternative).

\section{Application of the method}

Having outlined the concepts of egalitarianism and sufficientarianism and identified a method to incorporate them in quantitative evaluations of SRAIs, we next demonstrate the practical application of the method. The indicators are applied for three municipalities in the Netherlands. In The Hague, one of the country's large cities, with high urban densities, a high mix of uses, and almost entirely surrounded by urban area of other cities, services are nearby in all neighbourhoods. Dongeradeel, on the other hand, is a small town surrounded by countryside and small townships in the northern part of the country, where the level of services has been declining for decades. Both municipalities border to the sea. The third city, Delft, is a medium-sized city nearby The Hague, and partly surrounded by an urban area.

A measure of the accessibility of basic services was developed for every postal code area (which is more or less a neighbourhood). The chosen basic services are assumed to be necessary to prevent households from social exclusion, and include a greengrocer, butcher, 
baker, bookshop or stationer, drugstore, pharmacy, family doctor and dentist. The first five shops are replaced by a supermarket if this improves the accessibility.

Suppose that individuals at residential location $i$ need to have access to a set of $S$ services distributed over discrete points in space. The simplest accessibility measure, often referred to as a cumulative opportunity measure (Handy and Niemeier 1997), counts all services within a given travel distance, time or cost threshold. As this measure is highly sensitive to the distance range, it was improved by adding a distance decay factor, indicating that the farther away, the lower is the likelihood that the individual visits destinations. This measure was first used by Hansen (1959) and referred to as a gravity-based measure.

With both measures, a high value indicates that there are more services within reach, while they do not distinguish between service types. For example, the value rises with more grocery shops nearby even though a health clinic is too far away to access. However, for the purposes of social inclusion minimal necessary accessibility should be measured across a full range of different basic services. For this, the measure should include at least one occurrence of every type of these basic services, in such a way, that the minimal travel cost is that needed to reach each of them.

The most intuitive way is to allocate the nearest occurrence of each of the S services and totalise them. A shortcoming is that this assumes that individuals make only home-based visits, while in reality they are inclined to chain destinations to save travel time (Maat and Timmermans 2006). Generally, individuals will not just connect the most nearby destinations, but also take the distances between the destinations into account (Arentze et al. 1993). We assume that they aim to visit clusters of destinations rather than searching for the real shortest path between destinations.

To identify a cluster, we assume that the average distance between the closest services of each type indicates the compactness of a cluster. We calculate for each destination $j$, the shortest distance to each other $j$, whereby for every type of $s$, the occurrence with the shortest distance is allocated. Each residential location $i$ is allocated to a cluster on the basis of the shortest distance connecting $i$ with all services in a cluster.

In the next step, the Lorenz curve is derived for (all inhabitants of) each municipality on the basis of this cluster index. In addition, the cumulative opportunity measure, simply referred to as the buffer index, using a $2 \mathrm{~km}$ radius, is calculated. The Lorenz curve shows the distribution of total accessibility over the population. A point on a Lorenz curve shows the percentage $\mathrm{y}$ of total accessibility for the $\mathrm{x} \%$ of people with the lowest level of accessibility. The line of inequality is depicted by the diagonal. The Lorenz curve shows the deviation: further away from the diagonal represents higher levels of inequality.

The measure of egalitarianism is described by the Gini-index, which varies between 0 and 1, with higher coefficients representing higher levels of inequality. The measure of sufficientarianism is described by the slope of the Lorenz curve up to the threshold value.

Figure 2 below shows the Lorenz curve of Dongeradeel, The Hague and Delft, with the cluster index depicted on the left and the buffer index (using a $2 \mathrm{~km}$ radius) on the right. All the values were normalized for easy comparison. It appears that the small town of Dongeradeel deviates most from the 'line of equality', which is also evident from the Gini value (see Table 1), indicating less equality than in the bigger cities. The explanation is obvious, as households in cities have better access to daily services, while in Dongeradeel this only counts for inhabitants of the central town. The inhabitants of Dongeradeel's township have very low access to daily services. The inhabitants of the cities have much better and more equal access to the services. It is remarkable that Delft has even better 

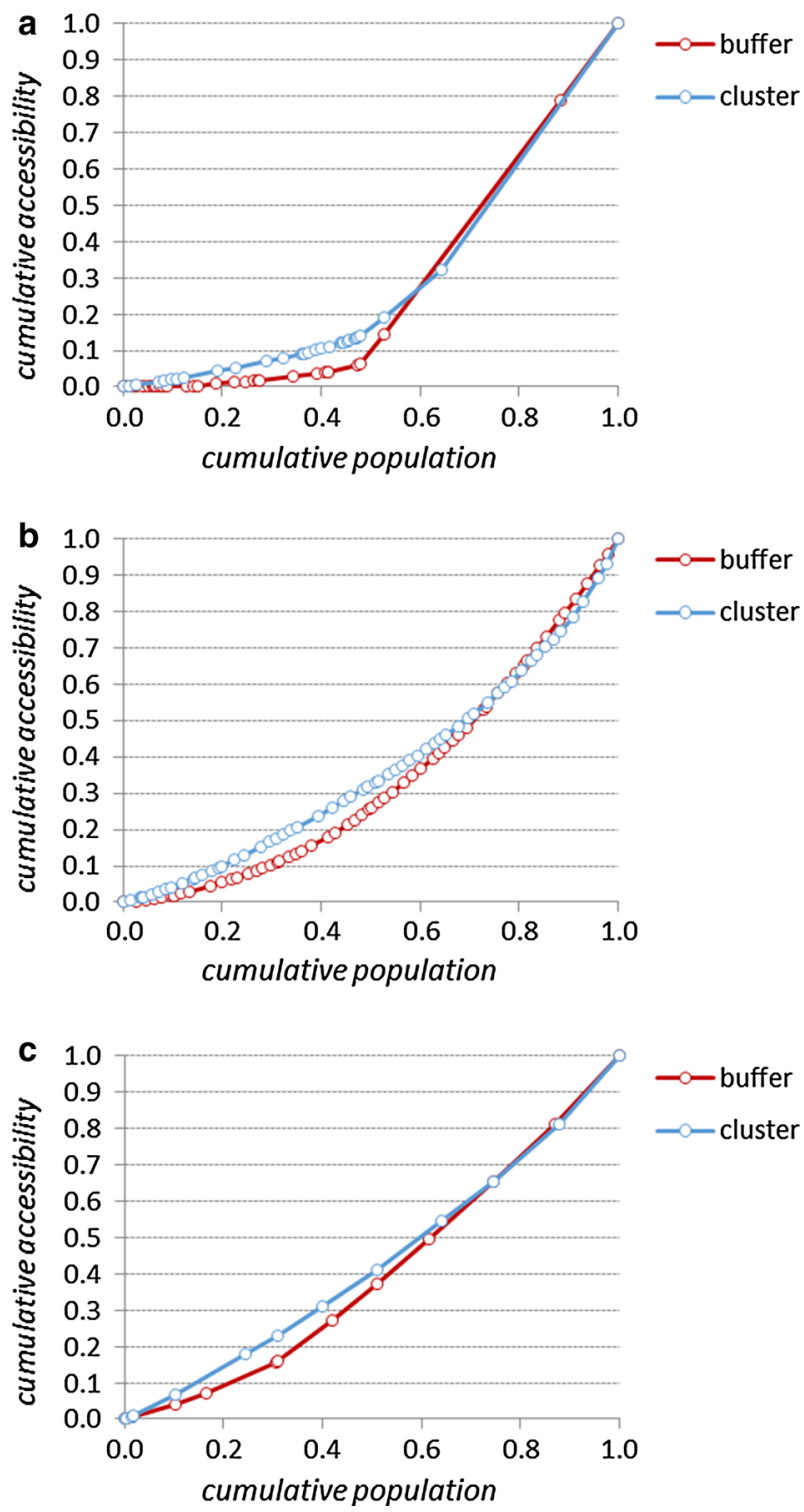

Fig. 2 a Lorenz curves of Dongeradeel. b Lorenz curves of The Hague. c Lorenz curves of Delft

access to the services than the bigger town of The Hague: in particular the neighbourhoods bordering the sea and the dunes have only one-sided access to services.

The curves and Gini-indices also show some differences between the cluster and the buffer indices: the buffer shows a little more inequality. This is plausible, as in low urbanised areas a basic service level will remain, with only one occurrence of every service type, while in high urbanised areas many occurrences are available, giving more choice.

The share of the population below a certain accessibility threshold is calculated for four thresholds of the cluster measure: the share of population that have access all basic services 
Table 1 Indices of egalitarianism and sufficientarianism

\begin{tabular}{lllc}
\hline & Dongeradeel & The Hague & Delft \\
\hline Gini cluster index & .65 & .27 & .13 \\
Gini buffer index & .70 & .33 & .20 \\
Threshold cluster index & Share of persons within threshold \\
$1 \mathrm{~km}$ & .00 & .82 & .49 \\
$2 \mathrm{~km}$ & .47 & 1.00 & 1.00 \\
$4 \mathrm{~km}$ & .53 & 1.00 & 1.00 \\
$5 \mathrm{~km}$ & .56 & 1.00 & 1.00 \\
Area As as percentage of triangle & & .02 & .08 \\
For thresholds value of $1 \mathrm{~km}$ & .65 & & \\
\hline
\end{tabular}

within 1, 2, 4 and $5 \mathrm{~km}$ travel, suggesting that those who have to travel over this threshold are socially excluded. According to this indicator, The Hague performs best, as its entire population has to travel $2 \mathrm{kms}$ at most to get their basic services, and $82 \%$ needs to travel no more than $1 \mathrm{~km}$. In Dongeradeel, on the contrary, about half of the population has to travel more than $5 \mathrm{kms}$.

For the threshold value of $1 \mathrm{~km}$ the area 'As' as a percentage of the triangle (area under the equal distribution line), an indicator for the severity of social exclusion, equals the Gini-index for Dongeradeel because all inhabitants are below this threshold value. For The Hague and Delft values equal .02 and .08 respectively, indicating an about a four times larger 'social exclusion problem' in Delft compared to The Hague.

Comparing the results of Table 1, we can conclude that in our case the comparison is mainly influenced by the threshold value of the cluster index. The value of $1 \mathrm{~km}$ results in most contrast between the three areas, both with respect to the share of the people being socially excluded, as well as the 'As' area as a fraction of the area below the equal distribution line (note we did not calculate values for other thresholds because no inhabitants in both The Hague and Delft are below the threshold values-see Table 1). Differences between The Hague and Delft disappear applying a threshold value of $2 \mathrm{~km}$ or more. The difference between Dongeradeel and The Hague and Delft is not very sensitive for the choice between 2,3 or $5 \mathrm{~km}$. In other words, in this case the choice for a method based on egalitarianism (Gini coefficients) versus sufficientarianism (share of persons within threshold) seems to be of less importance than the threshold value in the approach based on sufficientarianism.

\section{Critical discussion and implications of the SRAI method for future transport policy decision-making}

The above case study example demonstrates that if the data is available it is practicably possible to assess the extent to which different areas provide sufficient accessibility for social inclusion. We now present the arguments for why this is a relevant and valid method for contemporary transport policymaking.

Why should we value accessibility?

Accessibility is arguably linked to broader concepts of quality of life, happiness and wellbeing and academics elsewhere have argued that it is more relevant to evaluate SRAI 
policies in this wider context (Stanley and Vella-Brodrick 2009). For example, Stanley and Stanley (2007) argue that reducing social exclusion per se is not the ultimate policy goal, which should instead be expressed in terms of enhancing quality of life. We would not claim that improved accessibility will always lead to enhanced quality of life, but it can contribute to it significantly.

What types of transport policies should be evaluated for their SRAIs?

There are many examples of policy questions that could be evaluated with our method. For example, the impact of investments in high speed rail (HSR) versus rural or urban rail or bus systems on SRAI could be evaluated. A likely outcome would be that HSR has no impact on the level of social exclusion and increases the Gini-coefficient (in other words: increases differences in accessibility over (groups of) population). On the other hand, local rural or urban rail or bus systems probably will improve accessibility and so are more likely to reduce transport-related social exclusion. Another example could be the evaluation of walking and cycling policies on SRAI.

Do accessibility measures capture social inclusion well enough?

The ethically based accessibility approaches we have suggested implicitly assume that levels of accessibility are inherently related to levels of social exclusion. Whilst we believe this is partially the case, other important dimensions need to be considered. For example, we have explained elsewhere (Lucas 2012) this may not be so strongly the case in all instances, for three reasons:

1. People's social exclusion is not always or solely the results of a lack of access to destinations only, but of a complex set of factors, that inevitably are not all covered with in the accessibility indicator.

2. The new activities or locations people are being connected to are not suited to their needs, e.g. the jobs are not suitable for low-skilled workers; hospitals do not have with right clinics, colleges with the right courses, etc.

3. The targeted audience may not have the capacity (e.g. because of other responsibilities and other time constraints) and/or may be unwilling (e.g. because of the low return of the activity) to take advantage of the improved accessibility that is provided.

These are all issues for further exploration and research, which the constraints of this paper prevent us from discussing these in greater detail. We raise them here in the hope of furthering their discussion and debate within the transport discipline, as well as opening up further potentially fruitful lines of enquiry for research. Despite these caveats, we believe that our ethically based SRAI method is useful to gain ex ante indicative insights into the equity and inclusion implications of different policies measures. It is plausible to assume that the policies that score better in our method would also score better when applied in the 'real world'.

\section{Concluding remarks}

We have proposed that the two ethical theories of egalitarianism and sufficientarianism can provide the theoretical framing for the evaluation of SRAIs. We do not claim our proposals are the only option to include SRAIs but an important advantage is firstly that our 
proposals are well underpinned by ethical theories, and secondly that the Gini index is a well-known indicator to measure inequality. Ethics-driven methods may provide decision makers with a justifiable and, thus, more defensible policy approach.

We show that the method is applicable to real world cases, but as argued above we know that it needs further development. For practical applications we firstly think it is important that policy-makers determine exactly which activities and destinations are relevant for inclusion within the assessment: i.e. which activities and destination locations matter the most for the participation of which social groups. They will similarly need to determine appropriate threshold values for the levels of accessibility needed to secure their social inclusion.

Ideally, both the accessibility indicators and their threshold levels need to be identified in close collaboration with the intended audiences for improved accessibility measures, local policymakers and other key stakeholders. However, and especially for future levels of accessibility, it is often not feasible to interview people about their perceived future needs. To overcome this problem, they will need to establish to what extent the transport and travel experiences of socially excluded people can be correlated with more readily available standardised measures.

Methodologically more insight is needed into the role of data quality on the sensitivity of assessment outcomes. We also intend more research on the impact of disaggregation of the population over zones (how small should they be?). We realise that within the zones differences in accessibility exist. The smaller the threshold values used in case of sufficientarianism, the more important spatial disaggregation will become.

A question also remains on how to integrate SRAI assessment within traditional evaluation frameworks, like MCA and CBA. We argue the method we have presented in this paper can be relatively easily incorporated in an MCA but integration in a CBA framework may not be as possible. In that case, we would suggest that a monetary value should be derived expressing the valuation of changes in the indicators used. A possible solution could be to further develop the concept of the Social Welfare Functions for the area of SRAI, or to undertake further research into the Willingness To Pay for Fairness (WTPF) of both amongst politicians and the wider public. This is an indicator to value fairness at an aggregate level and diverges from the conventional Willingness To Pay of consumers, e.g. the Willingness To Pay for an additional trip (e.g. Stanley et al. 2011).

Acknowledgments We thank the four reviewers of our paper for their detailed and extremely useful comments on our earlier drafts, which have helped us to significantly improve the quality of our final submitted version.

Open Access This article is distributed under the terms of the Creative Commons Attribution License which permits any use, distribution, and reproduction in any medium, provided the original author(s) and the source are credited.

\section{References}

Arentze, T., Borgers, A., Timmermans, H.: A model of multi-purpose shopping trip behavior. Pap. Reg. Sci. 72(3), 239-256 (1993)

Arrow, K.J.: Social Choice and Individual Values, 2nd edn. Wiley, New York (1963)

Bristow, A., Nellthorp, J.: Transport project appraisal in the European Union. Trans. Policy 7(1), 51-60 (2000) 
Currie, G., Richardson, T., Smyth, P., Vella-Brodrick, D., Hine, J., Lucas, K., Stanley, J., Morris, J., Kinnear, R., Stanley, J.: Investigating links between transport disadvantage, social exclusion and wellbeing in Melbourne-preliminary results. Trans. Policy 16(3), 97-105 (2009)

Delbosc, A., Currie, G.: Using Lorenz curves to assess public transport equity. J. Trans. Geogr. 19(6), 1252-1259 (2011)

Department for Transport: Social exclusion and the provision and availability of public transport (2002). https://www.liftshare.com/business/pdfs/Dft-social\%20exclusion.pdf

Department for Transport: Accessibility planning: full guidance (2006). http://assets.dft.gov.uk/publications/ accessibility-planning-guidance/accessibility-planning-guidance-full-guidance.pdf

Department for Transport: Transport Analysis Guidance: WebTAGUnits A4.1 (2014). https://www.gov.uk/ government/uploads/system/uploads/attachment_data/file/370908/TAG_Unit_A4.1_-_Social_Impact_ Appraisal_January2014.pdf and A4.2 https://www.gov.uk/government/uploads/system/uploads/ attachment_data/file/370537/webtag-tag-unit-a4-2-distributional-impact-appraisal.pdf

Eijgenraam, C.J.J., Koopmans, C.C., Tang, P.J.G., Verster, A.C.P.: Evaluation of infrastructural projects; Guide for cost -benefit analysis, Sections I and II, CPB. NEI, Rotterdam, The Hague (2000)

Gardiner, S.M.: Protecting future generations: intergenerational buck-passing, theoretical eptitude and a brief for a global core precautionary principle. In: Tremmel, J.C. (ed.) Handbook of Intergenerational Justice. Edward Elgar, Chelterham, Northhampton (2006)

Geurs, K.T., van Wee, B.: Accessibility evaluation of land-use and transport strategies: review and research directions. J. Trans. Geogr. 12, 127-140 (2004)

Gini, C.: On the measure of concentration with special reference to income and statistics, Colorado College Publication, General Series No. 208, pp. 73-79 (1936)

González-Pérez, G.J., Vega-López, M.G., Romero-Valle, S., Vega-López, A., Cabrera-Pivaral, C.E.: A socio-spatial analysis of social exclusion and inequity in health in Mexico [Exclusión social e inequidadensaluden México: Unanálisis socio-espacial] Revista de Salud Publica. 10(1), 15-28 (2008)

Grant-Muller, S.M., MacKie, P., Nellthorp, J., Pearman, A.: Economic appraisal of European transport projects: the state-of-the-art revisited. Trans. Rev. 21(2), 237-261 (2001)

Handy, S., Niemeier, D.A.: Measuring accessibility: an exploration of issues and alternatives. Environ. Plan. A 29, 1175-1194 (1997)

Hayashi, Y., Morisugi, H.: International comparison of background concept and methodology of transportation project appraisal. Trans. Policy 7(1), 73-88 (2000)

Hansen, W.G.: How accessibility shapes land use. J. Am. Inst. Plan. 35(2), 73-76 (1959)

Hine, J., Mitchell, F.: Better for everyone? Travel experiences and transport exclusion. Urban Stud. 38(2), 319-332 (2001)

HM Treasury (2014) The Green Book: appraisal and evaluation in central government. https://www.gov.uk/ government/uploads/system/uploads/attachment_data/file/220541/green_book_complete.pdf

Hurni, A.: Transport and Social Exclusion in Western Sydney, University of Western Sydney and Western Sydney Community Forum, Australia (2006)

Hyard, A.: Cost-benefit analysis according to Sen: an application in the evaluation of transport infrastructures in France. Trans. Res. Part A 46(4), 707-719 (2012)

Jones, P., Lucas, K.: The social consequences of transport decision-making: clarifying concepts, synthesizing knowledge and assessing implications. J. Trans. Geogr. 21(2), 4-16 (2012)

Karlström, A., Franklin, J.P.: Behavioral adjustments and equity effects of congestion pricing: analysis of morning commutes during the Stockholm Trial. Trans. Res. Part A 43(3), 283-296 (2009)

Kelly, E. (ed.): Justice as Fairness: A Restatement. Harvard University Press, Cambridge (2001)

Litman, T.: The future isn't what it used to be: changing trends and their Implications for transport planning (2012). http://www.vtpi.org/future.pdf

Lucas, K.: Transport and social exclusion: Where are we now?. Trans. Policy (2012). (published online at http://dx.doi.org/10.1016/j.tranpol.2012.01.013)

Maat, K., Timmermans, H.: Influence of land use on tour-complexity, a Dutch case. Trans. Res. Record 1977, 234-241 (2006)

Mackett, R., Achuthan, K., Titheridge, H.: AMELIA: a tool to make transport policies more socially inclusive. Trans. Policy 15(6), 372-378 (2008)

Martens, K.: Justice in transport as justice in accessibility: applying Walter's 'Spheres of Justice' to the transport sector. Transportation (2012). doi: 10.1007/s11116-012-9388-7

Martens, K., Golub, A., Robinson, G.: A justice-theoretic approach to the distribution of transportation benefits: implications for transportation planning practice in the United States. Trans. Res. Part A 46(4), 684-695 (2012) 
Meyer, L.H., Roser, D.: Enough for the future. In: Gosseries, A., Meyer, L.H. (eds.) Intergenerational Justice. Oxford University Press, Oxford (2009)

Neutens, T., Schwanen, T., Witlox, F., Mayer, P.: Equity of urban service delivery: a comparison of different accessibility measures. Environ. Plan. A 42, 1613-1635 (2010)

Nyborg, K.: Homo economicus and homo politicus: interpretation and aggregation of environmental values. J. Econ. Behav. Org. 42(3), 305-322 (2000)

Páez, A., Mercado, R.G., Farber, S., Morency, C., Roorda, M.: Mobility and Social Exclusion in Canadian Communities: An Empirical Investigation of Opportunity Access and Deprivation from the Perspective of Vulnerable Groups. Policy Research Directorate Strategic Policy and Research, Toronto (2009)

Preston, J., Raje, F.: Accessibility, mobility and transport-related social exclusion. J. Trans. Geogr. 15(3), 151-160 (2007)

Ramjerdi, F.: Equity measures and their performance in transportation. Trans. Res. Record 1983, 67-74 (2006)

Rawls, J.: A Theory of Justice. Harvard University Press, Boston (1971)

Rietveld, P., Rouwendal, J., Van der Vlist, A.J.: Equity Issues in the Evaluation of Transport Policies and Transport Infrastructure Projects. In: van Geenhuizen, M., Reggiani, A., Rietveld, P. (eds.) Policy Analysis of Transport Networks, pp. 19-36. Ashgate, Aldershot (2007)

Sagoff, M.: The Economy of the Earth. Cambridge University Press, New York (1988)

Santos, B., Antunes, A., Miller, E.J.: Integrating equity objectives in a road network design model. Trans. Res. Record Issue 2089(1), 35-42 (2008)

Schwanen, T., Kwan, M.-P.: The Internet, mobile phone and space-time constraints. Geoforum 39(3), 1362-1377 (2008)

Sen, A.: Inequality Reexamined. Cambridge University Press, Cambridge (1992)

Sen, A.: The Idea of Justice. Allen Lane, London (2009)

Social Exclusion Unit: Making the Connections: Final report on Transport and Social Exclusion. Office of the Deputy Prime Minister, London (2003)

Stanley, J.R., Stanley, J.K.: Public transport and social policy goals. Road Trans. Res. 16(1), 20-30 (2007)

Stanley, J., Vella-Brodrick, D.: The usefulness of social exclusion to inform social policy in transport. Trans. Policy 16(3), 90-96 (2009)

Stanley, J., Hensher, D.A., Stanley, J., Currie, G., Greene, W.H., Vella-Brodrick, D.: Social Exclusion and the Value of Mobility. J. Trans. Econ. Policy 45(2), 197-222 (2011)

Thomopoulos, N., Grant-Muller, S., Tight, M.R.: Incorporating equity considerations in transport infrastructure evaluation: current practice and a proposed methodology. Eval. Progr. Plan. 32(4), 351-359 (2009)

Thomopoulos, N., Grant-Muller, S.: Incorporating equity as part of the wider impacts in transport infrastructure assessment: an application of the SUMINI approach. Transportation 40(2), 315-345 (2013)

Tian, Q., Huang, H.-J., Yang, H., Gao, Z.: Efficiency and equity of ramp control and capacity allocation mechanisms in a freeway corridor. Trans. Res. Part C: Emerg. Technol. 20(1), 126-143 (2012)

Young, W., Tilley, F.: Can businesses move beyond efficiency? The shift toward effectiveness and equity in the corporate sustainability debate. Bus. Strat. Environ. 15(6), 402-415 (2006)

Van Wee, B.: Rail infrastructure: challenges for cost-benefit analysis and other ex ante evaluations. Trans. Plan. Technol. 30(1), 31-48 (2007)

Van Wee, B.: Transport and Ethics. Ethics and The Evaluation of Transport Policies and Projects. Edward Elgar, Cheltenham (2011)

Van Wee, B., Geurs, K.T.: Discussing equity and social exclusion in accessibility evaluations. EJTIR 11(4), 350-367 (2011)

Van Wee, B.: How suitable is CBA for the ex-ante evaluation of transport projects and policies? A discussion from the perspective of ethics. Trans. Policy 19(1), 1-7 (2012)

Wagner, A., Hann, M., Noyce, P., Ashcroft, D.: Equity in the distribution of community pharmacies in England: impact of regulatory reform. J. Health Serv. Res. Policy 14(4), 243-248 (2009)

Weymark, J.A.: Generalized gini inequality indices. Math. Soc. Sci. 1(4), 409-430 (1981)

Wolf, C.: Climate change and climate policies. In: Gosseries, A., Meyer, L.H. (eds.) Intergenerational Justice. Oxford University Press, Oxford (2009) 
Karen Lucas is Associate Professor of Transport Geography and the Director of Research and Innovation at the Institute for Transport Studies at the University of Leeds, United Kingdom.

Bert van Wee is professor of Transport Policy at Delft University of Technology, the Netherlands, and scientific director of TRAIL research school.

Kees Maat is associate professor and head of the Urban and Regional Planning group at TU Delft. His research deals with the interaction between the built environment and travel behaviour. 